\title{
Stability analysis and synchronization of incommensurate fractional-order neural netwroks
}

Research Article

\section{Amel Hioual $^{1 *}$ and Taki-Eddine Oussaeif ${ }^{1 \dagger}$}

1 Departement of Mathematics and Computer sciences, University of Larbi Ben M'hidi, Oum El Bouaghi, Algeria.

\begin{abstract}
This paper develops a theoretical framework for analyzing the stability of nonlinear incommensurate fractionalorder neural networks. A necessary theorem for asymptotical stability is established using the characteristic equation for a nonlinear fractional-order system, and how to employ this theorem in stabilization is also presented. With the suitable control, the difficulties of stabilization and synchronization of fractional-order chaotic incommensurate fractional-order neural networks may be readily overcome. Two numerical examples have been shown to demonstrate how the established theory may be used to investigate stability and construct stabilization controllers.

Keywords: Fractional calculus • Incommensurate fractional-order neural networks • Asymptotic stability • Synchronization
\end{abstract}

\section{Introduction}

The study of differential and integral operators of real and complex order referred to as fractional calculus was developped by many famous mathematicians such as Liouville, Riemann, Abel, and CaputoEven though the branch of fractional calculus began practically concurrently with its integer-order equivalent, the mathematics and, in particular, its applications are somewhat less developed, and several causes have led to this conclusion. The fact that these operators are multiscale by nature is noteworthy, as a consequence, memory effects are enabled by time-fractional operators.In the last few decades, fractional calculus has developed fast and has many applications in a wide range of areas [13][8][2][18][1]. It has been revealed that fractional differential equations are strongly connected to systems having memory and hereditary features [20][21][11],Furthermore, it offers an

\footnotetext{
* E-mail: amel.hioual@univ-oeb.dz
}

$\dagger$ E-mail: taki_maths@live.fr 
efficient description approach for mathematical models, as an example secure communication [2], control fields [23], digital signal processor [17] and so on.

In recent years, neural networks have received a great deal of attention due to their effective applications in a wide range of fields, including optimization, function approximation, associative memory, signal processing, automated control, and etc [7][24][22]. These applications rely significantly on the stability qualities of neural networks. As a result, the stability analysis of neural networks has received much interest in the research. To its memory and heredity features, for that fractional-order neural networks are projected to be particularly useful in many applications [6]. It is also shown that fractional-order neural networks may be successfully employed in a variety of study domains [14][4].Strong requirements have been offered for commensurate fractional order neural networks (including derivation orders that are all multiples of the same order), in [27] Global asymptotic stability of fractional-order competitive neural networks with multiple time-varying-delay links was investigated, MittagLeffler stability and adaptive impulsive synchronization of fractional order neural networks in quaternion field was adressed in [3], and in [9], New criteria on finite-time stability of fractional-order hopfield neural networks with time delays was introduced, [15] treated $\alpha$-stability and $\alpha$-synchronization for fractional-order neural networks. On the other hand, the synchronization problem initially introduced in [19], is a basic phenomenon that has recently received a lot of attention, since it has both theoretical and practical implications. One of the key issues is to accomplish and explain synchronization of chaotic systems that are strictly distinct. As it has been demonstrated that certain neural networks can manifest chaotic behavior, different synchronization results and approaches regarding constant fractoinal order neural networks have been published in the previous decade [12][25][10][28][29][30]. Because of the complicated dynamics of incommensurate systems, these conclusions and approaches could not be simply extended and applied to the incommensurate case.

All of the articles mentioned above studied the commensurate fractional-order neural networks, which indicates that the conclusions achieved may be invalid for incommensurate fractional-order neural networks(this includes all derivation orders that are multiples of different order). Because commensurate fractional-order neural networks are a subtype of incommensurate fractional-order neural networks, it is crucial to explore the stability and synchronization of incommensurate fractional-order neural networks. However, relatively few related results concerning these systems have been reported so far. Motivated by the earlier discussion, in this work we address the stability and synchronization of incommensurate fractional-order neural networks.

This paper's reminder is arranged as follows. Section 2 contains some preliminaries, such as definitions, lemma and theorems to Caputo fractional-order calculation. In Section 3.1, numerous necessary conditions for determining the stability criteria of incommensurate fractional-order neural networks are developed. Some essential conditions for synchronization of incommensurate fractional-order neural networks are derived in Section 3.2. Section 4 provides two numerical simalations to demonstrate the efficacy of the stated results. Fanilly, Section 5 provides the conclusion to this study. 


\section{Preliminaries}

Definition 2.1 ([8]).

The fractional-order integral of order $\alpha$ for an integrable function $f:\left[t_{0},+\infty\right) \rightarrow \mathbb{R}$ is defined as

$$
t_{0} I_{t}^{\alpha} f(t)=\frac{1}{\Gamma(\alpha)} \int_{t_{0}}^{t}(t-\tau)^{\alpha-1} f(\tau) d \tau
$$

where $\alpha>0$, and $\Gamma($.$) is the Gamma function which is defined by$

$$
\Gamma(z)=\int_{0}^{\infty} e^{-t} t^{z-1} d t, \quad(\operatorname{Re}(z)>0
$$

where $\operatorname{Re}(z)$ is the real part of $z$.

Definition $2.2([8])$.

The caputo fractional-order derivative of order $\alpha$ for a function $f$ is defined as

$$
{ }_{t_{0}}^{C} D_{t}^{\alpha} f(t)=\frac{1}{\Gamma(n-\alpha)} \int_{t_{0}}^{t}(t-\tau)^{n-\alpha-1} f^{(n)}(\tau) d \tau
$$

where $t \geq t_{0}$ and $n$ is a positive integer such that $n-1<\alpha<n$.

particuly, when $0<\alpha<1$

$$
{ }_{t_{0}}^{C} D_{t}^{\alpha} f(t)=\frac{1}{\Gamma(1-\alpha)} \int_{t_{0}}^{t}(t-\tau)^{-\alpha} f^{\prime}(\tau) d \tau
$$

Proposition 2.1 ([8]). $\quad-{ }_{t_{0}}^{C} D_{t}^{\alpha} c=0$ holds, where $c$ is any constant.

- The linearity of the Caputo fractional order derivative:

$$
{ }_{t_{0}}^{C} D_{t}^{\alpha}\left(v_{1} f(t)+v_{2} g(t)=v_{1} \underset{t_{0}}{C} D_{t}^{\alpha} f(t)+v_{2} \underset{t_{0}}{C} D_{t}^{\alpha} g(t)\right.
$$

Lemma $2.1([8])$.

Let $n=[\alpha]+1$ for $\alpha$ or $n=\alpha$ for $\alpha \in \mathbb{N}_{+}$, if $x(t) \in C^{n}[a, b]$ then,

$$
{ }_{t_{0}}^{R L} I_{t}^{\alpha C} t_{0} D_{t}^{\alpha} x(t)=x(t)-\sum_{k=0}^{n-1} \frac{x^{(k)}(a)}{k !}\left(t-t_{0}\right)^{k}, \quad n-1<\alpha \leq n .
$$

In particular if $0<\alpha \leq 1$ and $x(t) \in C^{1}[a, b]$, then

$$
{ }_{t_{0}}^{R L} I_{t}^{\alpha} t_{0} D_{t}^{\alpha} x(t)=x(t)-x\left(t_{0}\right)
$$

Theorem 2.1 ([26]).

Consider the following n-dimensional linear fractional order system:

$$
{ }_{0}^{C} D_{t}^{\bar{\alpha}} x(t)=A x(t)
$$

where $\bar{\alpha}=\left(\alpha_{1}, \ldots, \alpha_{n}\right), \quad A=\left(a_{i j}\right)_{n * n}$ Assume $M$ be the lowest common multiple of the denominators ui's of $\alpha_{i}$ 's, where $\alpha_{i}=\frac{v_{i}}{u_{i}},\left(u_{i}, v_{i}\right)=1, u_{i}, v_{i} \in \mathbb{Z}^{+}$, for $i=1,2, \ldots, n$.

Then the zero solution of system (1) is globally asymptotically stable in the Lyapunov sense if all roots $\lambda_{i}$ 's of

$$
\operatorname{det}\left\{\operatorname{diag}\left(\left[\begin{array}{llll}
\lambda^{M \alpha_{1}} & \lambda^{M \alpha_{2}} & \ldots & \lambda^{M \alpha_{n}}
\end{array}\right]\right)-A\right\}=0
$$

satisfy $|\arg (\lambda)|>\frac{\pi}{2 M}$ 
Theorem $2.2([5])$.

Consider the following n-dimensional linear fractional order system:

$$
{ }_{0}^{C} D_{t}^{\bar{\alpha}} x(t)=f(x(t))
$$

where $\bar{\alpha}=\left(\alpha_{1}, \ldots, \alpha_{n}\right), f(x(t))=\left(f_{1}(x(t)), \ldots, f_{n}(x(t))\right.$ is a nonlinear continious function Assume $M$ be the lowest common multiple of the denominators ui's of $\alpha_{i}$ 's, where $\alpha_{i}=\frac{v_{i}}{u_{i}},\left(u_{i}, v_{i}\right)=1, u_{i}, v_{i} \in \mathbb{Z}^{+}$, for $i=1,2, \ldots, n$. Then the zero solution of system (2) is globally asymptotically stable in the Lyapunov sense if all roots $\lambda_{i}$ 's of

$$
\operatorname{det}\left\{\operatorname{diag}\left(\left[\begin{array}{llll}
\lambda^{M \alpha_{1}} & \lambda^{M \alpha_{2}} & \ldots & \lambda^{M \alpha_{n}}
\end{array}\right]\right)-J\right\}=0
$$

satisfy $|\arg (\lambda)|>\frac{\pi}{2 M}$

where $J=\left.\frac{\partial f}{\partial x}\right|_{x=x^{*}}$ is the jaconienne matrix of $f$

\section{Mains results}

The icommensurate fractional-order neural networks can be discrbed as follow

$$
\left\{\begin{array}{l}
{ }_{t_{0}}^{C} D_{t}^{\alpha_{1}} x_{1}(t)=-c_{1} x_{1}(t)+\sum_{j=1}^{n} b_{1 j} g_{j}\left(x_{j}(t)\right)+I_{1} \\
{ }_{t_{0}}^{C} D_{t}^{\alpha_{2}} x_{2}(t)=-c_{2} x_{2}(t)+\sum_{j=1}^{n} b_{2 j} g_{j}\left(x_{j}(t)\right)+I_{2} \\
\cdots \\
{ }_{t_{0}}^{C} D_{t}^{\alpha_{n}} x_{n}(t)=-c_{n} x_{n}(t)+\sum_{j=1}^{n} b_{1 j} g_{j}\left(x_{j}(t)\right)+I_{n} \\
x\left(t_{0}\right)=x_{0}
\end{array}\right.
$$

or it can be written as

$$
\left\{\begin{array}{l}
{ }_{t_{0}}^{C} D_{t}^{\bar{\alpha}} x(t)=-C x(t)+B g(x(t))+I \\
x\left(t_{0}\right)=x_{0}
\end{array}\right.
$$

where ${ }_{t_{0}}^{C} D_{t}^{\alpha_{i}}$, denotes the Caputo fractional derivative with order $\bar{\alpha}=\left(\alpha_{1}, \ldots, \alpha_{n}\right),\left(0<\alpha_{i}<1\right)$ and $n$ represents the number of units in a neural network. $x(t)=\left(x_{1}(t), x_{2}(t), \ldots, x_{n}(t)\right)^{t} \in \mathbb{R}$ is the state vector at time $t$. $\left(c_{i}\right) \in$ $\mathbb{R}^{n * n}, i \in\{1,2, \ldots, n\}$ with $c_{i}>0$ is a diagonal matrix referred to the self-feedback connection weight, $\left(b_{i j} \in \mathbb{R}^{n * n}\right.$ corresponds to the connection of ith neuron to the jth neuron at time $t . g: C\left(\left[t_{0}, T\right], \mathbb{R}^{n}\right) \rightarrow \mathbb{R}^{n}, g(t, x(t))=$ $\left(g_{1}(t, x(t)), g_{2}\left(t, x(t, x(t)), \ldots, g_{n}(t, x(t))\right)^{T}\right.$ is the neuron continous activation function and $I=\left(I_{1}, I_{2}, \ldots, I_{n}\right)^{T}$ is the external inputs vector.

\subsection{Stability of incommensurate fractional-order neural netwroks}

Theorem 3.1.

Suppose that $M$ is the lowest common multiple of the denominators $u_{i}$ 's of $\alpha_{i}$ 's, where $\alpha_{i}=\frac{v_{i}}{u_{i}}, \quad\left(u_{i}, v_{i}\right)=$ $1, u_{i}, v_{i} \in \mathbb{Z}^{+}$, for $i=1,2, \ldots, n$. the equilibrium point $x=x^{*}$ of system (3) is asymptotically stable if

$$
|\arg (\lambda)|>\frac{\pi}{2 M}
$$


for all roots $\lambda$ 's of the following equation

$$
\operatorname{det}\left\{\operatorname{diag}\left(\left[\begin{array}{llll}
\lambda^{M \alpha_{1}} & \lambda^{M \alpha_{2}} & \ldots & \lambda^{M \alpha_{n}}
\end{array}\right]\right)-(-C+B J)\right\}=0
$$

where

$$
J=\left.\frac{\partial g}{\partial x}\right|_{x=x^{*}}, \quad g=\left[g_{1}, g_{2}, \ldots, g_{n}\right]^{T}
$$

Proof. Solving the following equations yields the equilibrium points of system (3):

$$
-c_{i} x_{i}(t)+\sum_{j=1}^{n} b_{i j} g_{j}\left(t, x_{j}(t)\right)+I_{i}=0, \quad i=1,2, \ldots, n
$$

Suppose that $x^{*}=\left(x_{1}^{*}, x_{2}^{*}, \ldots, x_{n}^{*}\right)$ is an equilibrium point of system (3), i.e.

$$
-c_{i} x_{i}^{*}+\sum_{j=1}^{n} b_{i j} g_{j}\left(t, x_{j}^{*}\right)+I_{i}=0 ; \quad i=1,2, \ldots, n
$$

To analyze this point's asymptotic stability, we establish

$$
\kappa_{i}=x_{i}-x_{i}^{*}, \quad i=1,2, \ldots, n
$$

then we have

$$
-_{0}{ }^{C} D_{t}^{\alpha}\left(\kappa_{i}(t)+x_{i}^{*}\right)=-c_{i}\left(\kappa_{i}(t)+x_{i}^{*}\right)+\sum_{j=1}^{n} b_{i j} g_{j}\left(t,\left(\kappa_{j}\left((t)+x_{j}^{*}\right)\right)+I_{i}=0, \quad i=1,2, \ldots, n\right.
$$

which implies according to Lemma(2.1)

$$
{ }_{t_{0}}^{C} D_{t}^{\alpha_{i}} \kappa_{i}(t)=-c_{i}\left(\kappa_{i}(t)+x_{i}^{*}(t)\right)+\sum_{j=1}^{n} b_{i j} g_{j}\left(t,\left(\kappa_{j}\left((t)+x_{j}^{*}(t)\right)\right)+I_{i}=0, \quad i=1,2, \ldots, n\right.
$$

If function $f_{i}$ has second continuous partial derivatives in a ball $\mathbb{R}^{n}$ centered at point $x^{*}$, then we obtain

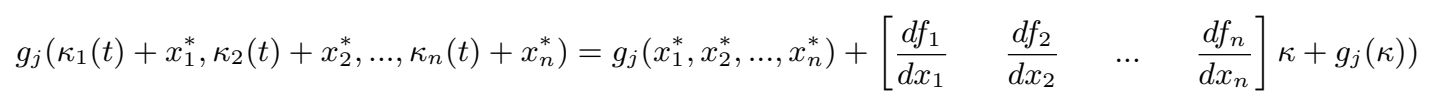

therefor (6) becomes

$$
{ }_{t_{0}}^{C} D_{t}^{\alpha_{i}} \kappa_{i}(t)=-c_{i}\left(\kappa_{i}(t)+x_{i}^{*}(t)\right)+\sum_{j=1}^{n} b_{i j}\left\{g_{j}\left(x_{j}^{*}(t)\right)+\frac{d f_{j}}{d x_{j}} \kappa_{j}(t)\right\}+I_{i}
$$

which gives using (5)

$$
{ }_{t_{0}}^{C} D_{t}^{\alpha_{i}} \kappa_{i}(t)=-c_{i} \kappa_{i}(t)+\sum_{j=1}^{n} b_{i j} \frac{d f_{j}}{d x_{j}} \kappa_{j}(t)
$$


and then

$$
\left[\begin{array}{c}
{ }_{t_{0}}^{C} D_{t}^{\alpha_{1}} \kappa_{1}(t) \\
{ }_{t_{0}}^{C} D_{t}^{\alpha_{2}} \kappa_{2}(t) \\
\ldots \\
{ }_{t_{0}}^{C} D_{t}^{\alpha_{n}} \kappa_{n}(t)
\end{array}\right]=(-C+B J) \kappa
$$

where

$$
J=\frac{d g}{d x}, \quad g=\left[g_{1}, g_{2}, \ldots, g_{n}\right]^{T}
$$

Suppose that $M$ is the lowest common multiple of the denominators $u_{i}$ 's of $\alpha_{i}$ 's, where $\alpha_{i}=\frac{v_{i}}{u_{i}}, \quad\left(u_{i}, v_{i}\right)=$ $1, u_{i}, v_{i} \in \mathbb{Z}^{+}$, for $i=1,2, \ldots, n$. According to Theorem 2.2, the equilibrium point $x=x^{*}$ of system (3) is asymptotically stable if

$$
|\arg (\lambda)|>\frac{\pi}{2 M}
$$

for all roots $\lambda$ 's of the following equation

$$
\operatorname{det}\left\{\operatorname{diag}\left(\left[\begin{array}{llll}
\lambda^{M \alpha_{1}} & \lambda^{M \alpha_{2}} & \ldots & \lambda^{M \alpha_{n}}
\end{array}\right]\right)-(-C+B J)\right\}=0
$$

Which completes the proof.

\subsection{Synchronization of incommensurate fractional order neural networks}

In this section, the synchronization of incommensurate fractional order neural networks is investigated.

As known a required condition for a fractional order system ${ }_{0}^{C} D_{t}^{\alpha} x(t)=f(x(t))$ to display the chaotic attractor is mathematically equal to:

$$
\frac{\pi}{2 M}-\min _{i}\left\{\left|\arg \left(\lambda_{i}\right)\right|\right\} \geq 0,
$$

where $\lambda_{i}$ 's are roots of equations:

$$
\operatorname{det}\left\{\operatorname{diag}\left(\left[\begin{array}{llll}
\lambda^{M \alpha_{1}} & \lambda^{M \alpha_{2}} & \ldots & \lambda^{M \alpha_{n}}
\end{array}\right]\right)-\frac{d f}{d x}\right\}=0
$$

Thus, under the following essential condition, incommensurate fractional-order neural networks (3) exhibit chaotic behavior if

$$
\frac{\pi}{2 M}-\min _{i}\left\{\left|\arg \left(\lambda_{i}\right)\right|\right\} \geq 0,
$$

where $\lambda_{i}$ 's are roots of equations:

$$
\operatorname{det}\left\{\operatorname{diag}\left(\left[\begin{array}{llll}
\lambda^{M \alpha_{1}} & \lambda^{M \alpha_{2}} & \ldots & \lambda^{M \alpha_{n}}
\end{array}\right]\right)-(-C+B J)\right\}=0
$$


Now, we consider a master system represented in (3) and a slave system given by:

$$
\left\{\begin{array}{l}
{ }_{0}^{C} D_{t}^{\alpha_{1}} y_{1}(t)=-c_{1} y_{1}(t)+\sum_{j=1}^{n} b_{1 j} g_{j}\left(y_{j}(t)\right)+I_{1}+U_{1}(t) \\
{ }_{0}^{C} D_{t}^{\alpha_{2}} y_{2}(t)=-c_{2} y_{2}(t)+\sum_{j=1}^{n} b_{2 j} g_{j}\left(y_{j}(t)\right)+I_{2}+U_{2}(t) \\
\cdots \\
{ }_{0}^{C} D_{t}^{\alpha_{n}} y_{n}(t)=-c_{n} y_{n}(t)+\sum_{j=1}^{n} b_{n j} g_{j}\left(y_{j}(t)\right)+I_{n}+U_{n}(t)
\end{array}\right.
$$

Definition 3.1 ([25]).

If any solutions $x(t)$ of $(3)$ and $z(t)$ of (7) satisfy the condition

$$
\lim _{t \rightarrow+\infty}\|x(t)-y(t)\|=0
$$

then systems (3) and (7) are said to be global asymptotic synchronization.

\section{Theorem 3.2.}

The master-slave pair (3)-(7) is globally complete synchronized by means of the control law:

$$
U_{i}(t)=-\left[\left(-c_{i}+a_{i}\right) e_{i}(t)-c_{i} e_{i}(t)+\sum_{j=1}^{n} b_{i j}\left(g_{j}\left(y_{j}(t)\right)-g_{j}\left(x_{j}(t)\right)\right)\right], \quad i=1, \ldots, n
$$

subject to the selection of the control matrix $A$ as follows

$$
A=\operatorname{diag}\left(\left[\begin{array}{lll}
a_{1} & \ldots & a_{n}
\end{array}\right]\right), \quad \text { such that }-a_{i}+c_{i}>0, \quad i=, \ldots, n
$$

Proof. Let's consider the following error

$$
e_{i}(t)=y_{i}(t)-x_{i}(t), \quad i=1, \ldots, n
$$

The Caputo fractional differential of the error system is given by

$$
{ }_{0}^{C} D_{t}^{\alpha_{i}} e_{i}(t)={ }_{0}^{C} D_{t}^{\alpha_{i}} y_{i}(t)-{ }_{0}^{C} D_{t}^{\alpha_{i}} x_{i}(t)=-c_{i} e_{i}(t)+\sum_{j=1}^{n} b_{i j}\left(g_{j}\left(y_{j}(t)\right)-g_{j}\left(x_{j}(t)\right)\right)+U_{i}
$$

Synchronization between master system (3) and slave system (7) is equivalent to the asymptotic stability of error system (8) with the suitable control law $U(t)$.

With the choose of

$$
U_{i}=-\left[\left(-c_{i}+a_{i}\right) e_{i}(t)-c_{i} e_{i}(t)+\sum_{j=1}^{n} b_{i j}\left(g_{j}\left(y_{j}(t)\right)-g_{j}\left(x_{j}(t)\right)\right)\right]
$$

we obtain

$$
{ }_{0}^{C} D_{t}^{\alpha_{i}} e_{i}(t)=\left(c_{i}-a_{i}\right) e_{i}(t)
$$

Since ${ }_{0}^{C} D_{t}^{\alpha_{i}} e_{i}(t)=\left(c_{i}-a_{i}\right) e_{i}(t)$ is a linear system according to Theorem (2.1) this system is stable iff

$$
\operatorname{det}\left\{\operatorname{diag}\left(\left[\begin{array}{llll}
\lambda^{M \alpha_{1}} & \lambda^{M \alpha_{2}} & \ldots & \lambda^{M \alpha_{n}}
\end{array}\right]\right)-(C-A)\right\}=0
$$

therefor $-c_{i}+a_{i}>0, \quad i=1, \ldots, n$

which accomplishes the proof. 


\section{Numerical examples}

All simulations in this section were run using Adams-Bashforth-Moulton algorithm described in [16] to solve a Caputo definition-based fractional differential equation.

Example 1 We consider the incommensurate fractional-order neural networks

$$
\left\{\begin{array}{l}
{ }_{0}^{C} D_{t}^{\alpha_{1}} x_{1}(t)=-c_{1} x_{1}(t)+b_{11} \tanh \left(x_{1}(t)\right)+b_{12} \tanh \left(x_{2}(t)\right)+I_{1} \\
{ }_{0}^{C} D_{t}^{\alpha_{2}} x_{2}(t)=-c_{2} x_{2}(t)+b_{21} \tanh \left(\frac{x_{1}(t)}{4}\right)+b_{22} \tanh \left(\frac{x_{2}(t)}{4}\right)+I_{2}
\end{array}\right.
$$

where

$$
B=\left[\begin{array}{ll}
-0.5 & 0.3 \\
-0.7 & 0.1
\end{array}\right] ; \quad C=\operatorname{diag}\left(\left[\begin{array}{ll}
0.1 & 0.2
\end{array}\right]\right) ; \quad I=\left(\begin{array}{ll}
0 & 0
\end{array}\right)^{T}
$$

and $\left(\alpha_{1}, \alpha_{2}\right)=(0.4,0.3)$

To obtain the equilibrium points, let us consider the equations as

$$
\left\{\begin{array}{l}
-0.1 x_{1}(t)-0.5 \tanh \left(x_{1}(t)\right)+0.3 \tanh \left(x_{2}(t)\right)=0 \\
-0.2 x_{2}(t)-0.7 \tanh \left(\frac{x_{1}(t)}{4}\right)+0.1 \tanh \left(\frac{x_{2}(t)}{4}\right)=0
\end{array}\right.
$$

After solving equations (10), we get the equilibrium point as $E(0,0)$.

The Jacobian matrix for the function $g(x(t))=\left[\tanh (x(t)), \tanh \left(\frac{x(t)}{4}\right)\right]^{T}$ is given as

$$
J=\left[\begin{array}{cc}
\frac{1}{\cosh ^{2}\left(x_{1}(t)\right)} & \frac{1}{\cosh ^{2}\left(x_{1}(t)\right)} \\
\frac{1}{4 \cosh ^{2}\left(x_{1}(t)\right)} & \frac{1}{4 \cosh ^{2}\left(x_{1}(t)\right)}
\end{array}\right]
$$

at the equilibrium point $E(0,0)$ the characteristic polynomial of the matrix $-C+B J$ is given by

$$
\Delta(\lambda)=\lambda^{7}+0.65 \lambda^{4} 0.525 \lambda^{3}+0.5325
$$

The roots $\lambda_{i}$ 's and their appropriate arguments of polynomial of (11) calculated below

$$
\begin{aligned}
& \lambda_{1,2}=0.5964+0.8154 i, \quad\left|\arg \left(\lambda_{1}\right)\right|=0.9393 \\
& \lambda_{3}=-0.8112+0.0000 i, \quad\left|\arg \left(\lambda_{3}\right)\right|=3.1416 \\
& \lambda_{4,5}=-0.5589+0.4965 i, \quad\left|\arg \left(\lambda_{4}\right)\right|=2.4153 \\
& \lambda_{6,7}=0.3681+0.4703 i, \quad\left|\arg \left(\lambda_{6}\right)\right|=0.9067
\end{aligned}
$$




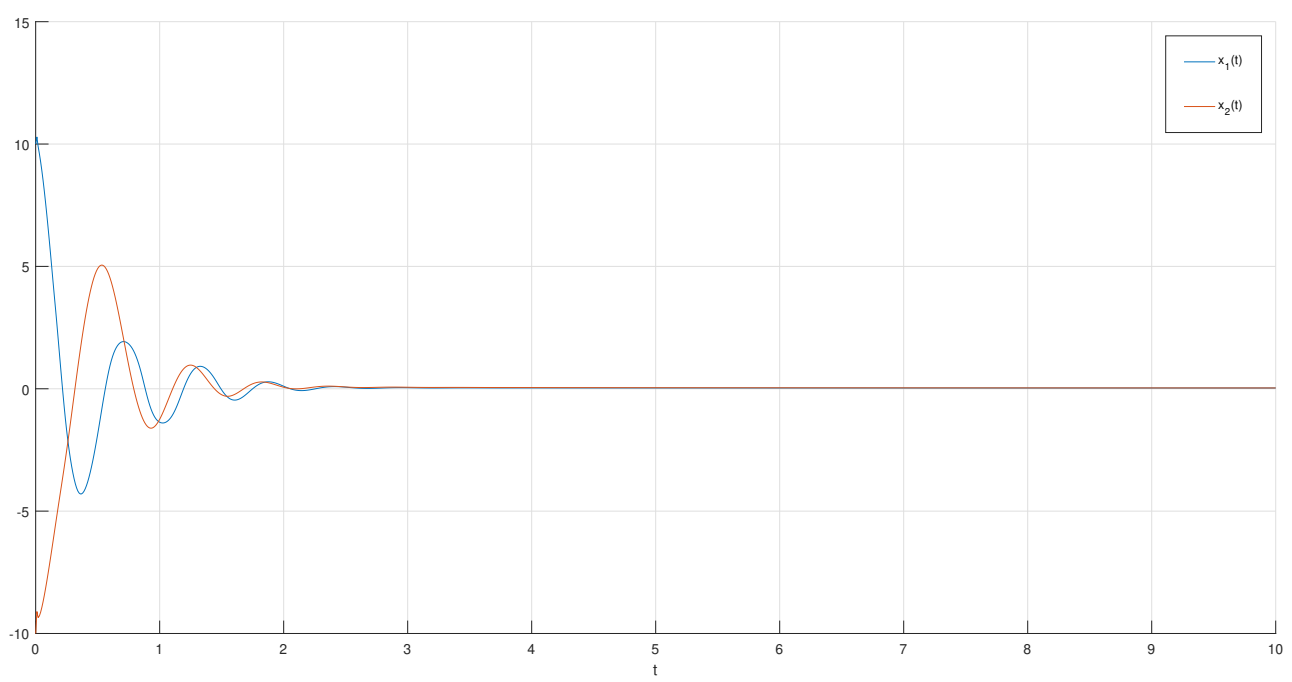

Figure 1. Numerical solution of the incommensurate neorder neural networks (9)

Since

$$
\left|\arg \left(\lambda_{i}\right)\right|>\frac{\pi}{2 M}=\frac{\pi}{20}
$$

The incommensurate neural networks (9) is asymptotically stable.

For time step size 0.01 and initial value $x_{0}=[10,-10]^{T}$, numerical simulation results in Figure 1 illustrate the asymptotically stability of system (9) for the given fractional-orders.

Exemple 2 Let's consider the three dimensionelle incommensurate fractional-order neural networks

$$
\left\{\begin{array}{l}
{ }_{0}^{C} D_{t}^{\alpha_{1}} x_{1}(t)=-c_{1} x_{1}(t)+b_{11} g\left(x_{1}(t)\right)+b_{12} g\left(x_{2}(t)\right)+b_{13} g\left(x_{3}(t)\right)+I_{1} \\
{ }_{0}^{C} D_{t}^{\alpha_{2}} x_{2}(t)=-c_{2} x_{2}(t)+b_{21} g\left(x_{1}(t)\right)+b_{22} g\left(x_{2}(t)\right)+b_{23} g\left(x_{3}(t)\right)+I_{2} \\
{ }_{0}^{C} D_{t}^{\alpha_{3}} x_{3}(t)=-c_{3} x_{3}(t)+b_{31} g\left(x_{1}(t)\right)+b_{32} g\left(x_{2}(t)\right)+b_{33} g\left(x_{3}(t)\right)+I_{3}
\end{array}\right.
$$

where

$$
B=\left[\begin{array}{ccc}
-1 & 3 & 2 \\
1 & -4 & 1 \\
5 & 1 & 2
\end{array}\right] ; \quad C=\operatorname{diag}(0.80 .80 .8) ; \quad I=\left[\begin{array}{lll}
0.01 & 0.01 & 0.01
\end{array}\right]
$$

$g(x(t))=\sin (x(t))$ the initial condition $x_{0}=(0.1,0.1,0.1)^{T}$ and $\left(\alpha_{1}, \alpha_{2}, \alpha_{3}\right)=\left(\frac{1}{3}, \frac{2}{3}, \frac{1}{3}\right)$ The equilibrium point is $x^{*}(0.0010,0.0027,0.0019)^{T}$ the characteristic polynomial of $-C+B J$ matrix is given by

$$
\Delta(\lambda)=\lambda^{4}-10.4 \lambda^{3}-6.16 \lambda^{2}-5.12 \lambda-5.888
$$



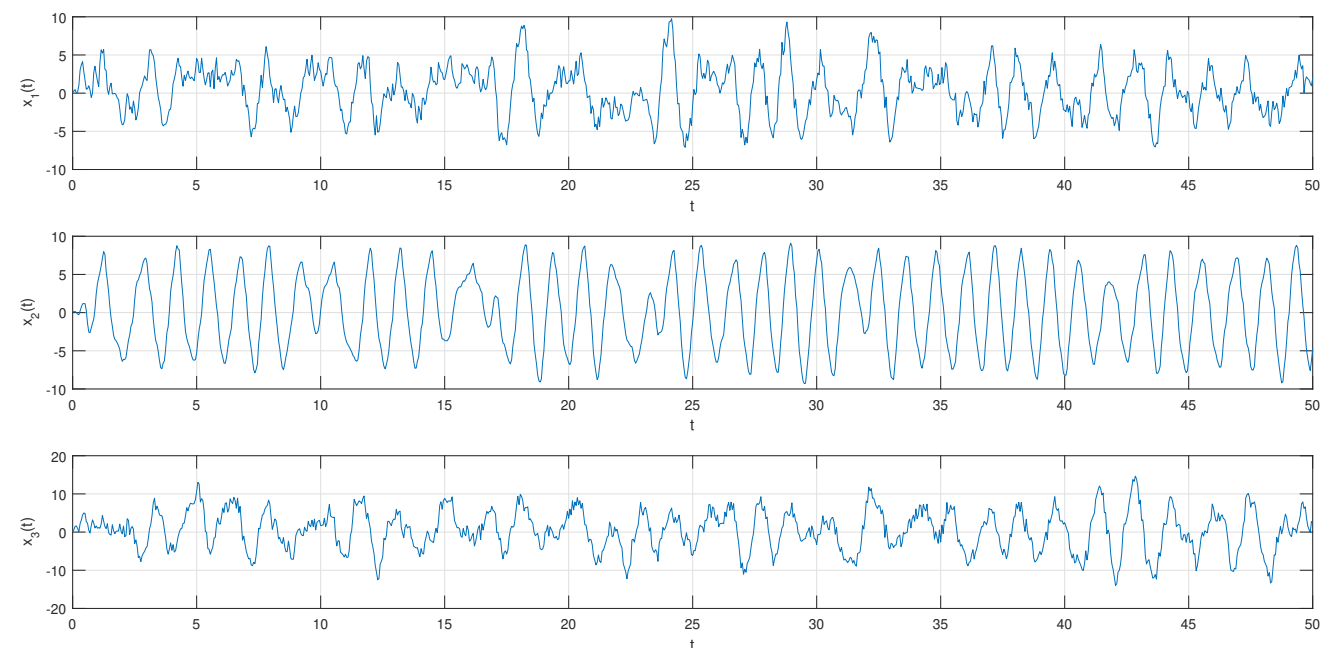

Figure 2. Chaotic behavor of the incommensurate fractional-order neural networks (12)

then the roots $\lambda$, and their appropriate arguments of polynomial (13) are

$$
\begin{gathered}
\lambda_{1}=11.0064+0.0000 i, \quad\left|\arg \left(\lambda_{1}\right)\right|=0 \\
\lambda_{2}=-0.8000+0.0000 i, \quad\left|\arg \left(\lambda_{3}\right)\right|=3.1416 \\
\lambda_{3,4}=0.0968+0.8120 i, \quad\left|\arg \left(\lambda_{4}\right)\right|=1.4521
\end{gathered}
$$

$x^{*}$ is unstable for the given fractional-orders because the eigenvalue $\lambda_{1}$ is a positive real integer. system (12) is considered the master system. Now, Let's consider the slave system

$$
\left\{\begin{array}{l}
{ }_{0}^{C} D_{t}^{\alpha_{1}} y_{1}(t)=-c_{1} y_{1}(t)+b_{11} g\left(x_{1}(t)\right)+b_{12} g\left(y_{2}(t)\right)+b_{13} g\left(y_{3}(t)\right)+I_{1}+U_{1} \\
{ }_{0}^{C} D_{t}^{\alpha_{2}} y_{2}(t)=-c_{2} y_{2}(t)+b_{21} g\left(y_{1}(t)\right)+b_{22} g\left(y_{2}(t)\right)+b_{23} g\left(y_{3}(t)\right)+I_{2}+U_{2} \\
{ }_{0}^{C} D_{t}^{\alpha_{3}} y_{3}(t)=-c_{3} y_{3}(t)+b_{31} g\left(y_{1}(t)\right)+b_{32} g\left(y_{2}(t)\right)+b_{33} g\left(y_{3}(t)\right)+I_{3}+U_{3}
\end{array}\right.
$$

Theorem 3.2 states that there exists a control matrix $A$ such that $C$ and $A$ fulfills the condition. One can, for example, select the form

$$
A=\operatorname{diag}\left(\left[\begin{array}{lll}
1.75 & 1.6 & 0.7
\end{array}\right]\right)
$$

This obviously meets the condition, as a result, systems (12) and (14) are synchronized. It is now quite 

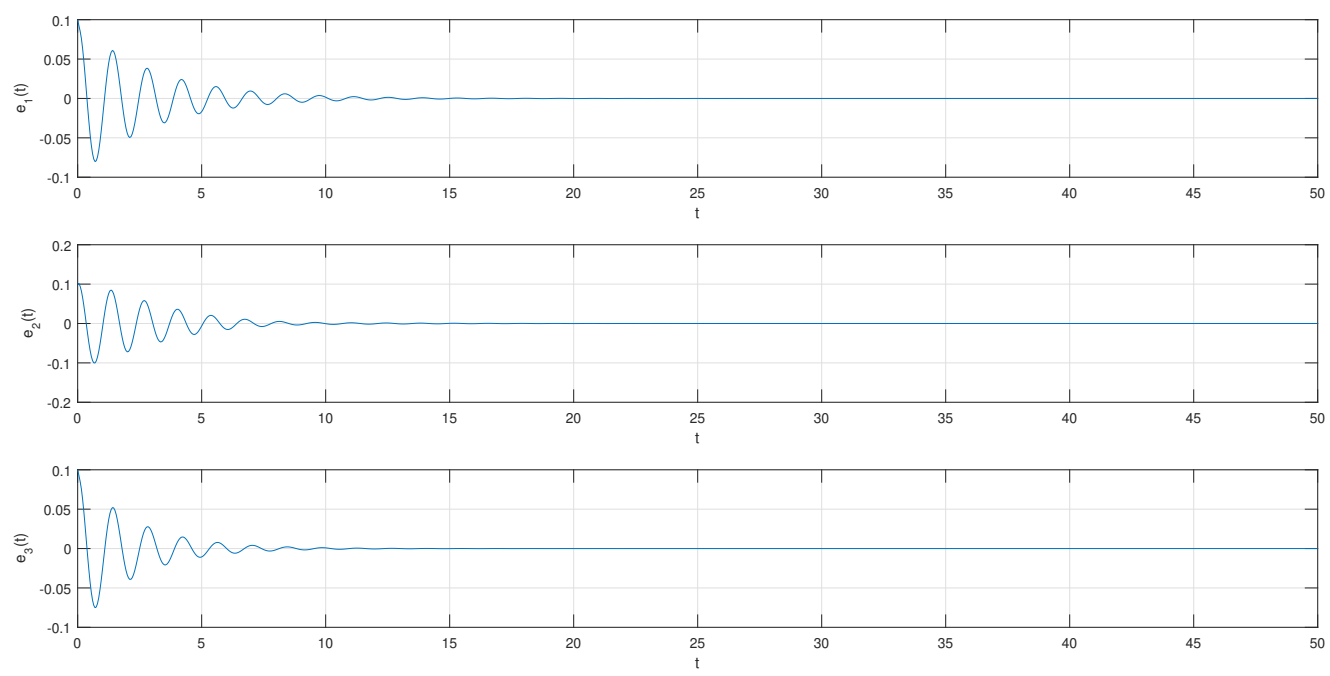

Figure 3. The evolution of the error system's states

simple to create the control law using Theorem 3.2. The final error system looks such as this

$$
\left\{\begin{array}{l}
{ }_{0}^{C} D_{t}^{\alpha_{1}} e_{1}(t)=-0.95 e_{1}(t) \\
{ }_{0}^{C} D_{t}^{\alpha_{2}} e_{2}(t)=-0.8 e_{2}(t) \\
{ }_{0}^{C} D_{t}^{\alpha_{3}} e_{3}(t)=-0.9 e_{3}(t)
\end{array}\right.
$$

Figure 3 depicts the temporal development of the errors. Clearly, synchronization is accomplished when the errors converge to zero in a reasonable amount of time.

\section{Conclusion}

The stability of incommensurate fractional-order neural networks is investigated in this research. Several interesting stability criteria are obtained from the introduced characteristic equation. We successfully derive a suitable stability requirement for incommensurate fractional-order neural networks using the data obtained. We also extend our conclusions to the synchronization of chaotic incommensurate fractional-order neural networks. Two numerical examples have been provided to illustrate the effectiveness of the theoretical analysis. 


\section{References}

[1] A. Boulkroune, A. Boubellouta. Chaos synchronization of optical systems via a fractional-order sliding mode controller. In Advanced Synchronization Control and Bifurcation of Chaotic Fractional-Order Systems, 218260. IGI Global, 2018. doi:10.4018/978-1-5225-5418-9. ch008.

[2] A.A. Kilbas, H.M. Srivastava, J.J. Trujillo . Theory and applications of fractional differential equations, Elsevier, 2006.

[3] A. Pratap, R. Raja, J. Alzabut, J. Cao. Mittag-leffler stability and adaptive impulsive synchronization of fractional order neural networks in quaternion field. Mathematical Methods in the Applied Sciences, 43(2020), no. 10, 6223-6253. https://doi.org/10.1002/mma.6367.

[4] A. Boroomand, M.B. Menhaj. Fractional-based approach in neural networks for identification problem. In 2009 Chinese Control and Decision Conference, 2319-2322. IEEE, 2009. doi: 10.1109/CCDC.2009.5192579.

[5] B. Daşbaşı . Stability analysis of an incommensurate fractional-order sir model. Mathematical Modelling and Numerical Simulation with Applications, 1(2021), no. 1. doi: 10.53391/mmnsa.2021.01.005.

[6] B.N. Lundstrom, M.H. Higgs, W.J. Spain, A.L. Fairhall. Fractional differentiation by neocortical pyramidal neurons. Nature neuroscience, 11(2008), no. 11, 1335-1342. https://doi.org/10.1038/nn.2212.

[7] C.T. Lin, M. Prasad, A. Saxena. An improved polynomial neural network classifier using real-coded genetic algorithm. IEEE Transactions on Systems, Man, and Cybernetics: Systems, 45, no. 11, 1389-1401. doi: 10.1109/TSMC. 2015.2406855.

[8] C. Kou, H. Zhou, Y. Yan. Existence of solutions of initial value problems for nonlinear fractional differential equations on the half-axis. Nonlinear Analysis: Theory, Methods \& Applications, 74(2011), no. 17, 5975-5986. https://doi.org/10.1016/j.na.2011.05.074.

[9] F. Du, J.G. Lu . New criteria on finite-time stability of fractional-order hopfield neural networks with time delays. IEEE Transactions on Neural Networks and Learning Systems, 2020. doi: 10.1109/TNNLS.2020. 3016038.

[10] F. Du, J.G. Lu. New criterion for finite-time synchronization of fractional order memristor-based neural networks with time delay. Applied Mathematics and Computation, 389(2021), 125616. https://doi.org/ $10.1016 / j$.amc.2020.125616.

[11] H. Delavari, M. Mohadeszadeh. Robust finite-time synchronization of nonidentical fractional-order hyperchaotic systems and its application in secure communication. IEEE/CAA Journal of Automatica Sinica, 6(2016), no. 1, 228-235. doi: 10.1109/JAS.2016.7510145.

[12] H. Wu, L. Wang, Y. Wang, P. Niu, B. Fang. Global mittag-leffler projective synchronization for fractionalorder neural networks: an lmi-based approach. Advances in Difference Equations, 2016(2016), no. 1, 1-18. https://doi.org/10.1186/s13662-016-0857-8. 
[13] I. Podlubny. Fractional differential equations: an introduction to fractional derivatives, fractional differential equations, to methods of their solution and some of their applications. Elsevier, 1998.

[14] J. Hu, J. Zhou, Y. Pu, Y. Liu, Y. Zhang. A novel fraction-based hopfield neural networks. In Soft Computing in Information Communication Technology, 143-150. Springer, 2012.

[15] J. Yu, C. Hu, H. Jiang. -stability and -synchronization for fractionalorder neural networks. Neural Networks, 35(2012), 82-87. https://doi.org/10.1016/j.neunet.2012.07.009.

[16] K. Diethelm, N.J. Ford, A.D. Freed . Detailed error analysis for a fractional adams method. Numerical algorithms, 36(2004), no. 1, 31-52. https://doi.org/10.1023/B:NUMA.0000027736.85078.be.

[17] L. Qiao, W. Zhang. Double-loop integral terminal sliding mode tracking control for uuvs with adaptive dynamic compensation of uncertainties and disturbances. IEEE Journal of Oceanic Engineering, 44(2018), no. 1, 29-53. doi: 10.1109/JOE.2017.2777638.

[18] M. Bettayeb, U.M. Al-Saggaf. High gain observer design for fractional-order non-linear systems with delayed measurements: application to synchronisation of fractional-order chaotic systems. IET Control Theory Applications, 11(2017), no. 17, 3171-3178. doi: 10.1049/iet-cta.2017.0396. .

[19] L.M. Pecora, T.L. Carroll. Synchronization in chaotic systems. Phys. Rev. Lett. 64(1990), no. 8, 821-824. https://doi.org/10.1103/PhysRevLett.64.821.

[20] R. Hilfer. Fractional calculus and regular variation in thermodynamics. In Applications of fractional calculus in physics, 429-463. World Scientific, 2000.

[21] R.L. Magin. Fractional calculus in bioengineering, volume 2. Begell House Redding, 2006.

[22] S. Yang, Z. Guo, J. Wang. Robust synchronization of multiple memristive neural networks with uncertain parameters via nonlinear coupling. IEEE Transactions on Systems, Man, and Cybernetics: Systems, 45(2015), no. 7, 1077-1086. doi: 10.1109/TSMC.2014.2388199.

[23] S.Y. Chen, C.Y. Lee. Digital signal processor based intelligent fractionalorder sliding-mode control for a linear voice coil actuator. IET Control Theory Applications, 11(2017), no. 8, 1282-1292. doi: 10.1049/iet-cta. 2016.1127.

[24] S. Wen, Z. Zeng, T. Huang, Q. Meng. Lag synchronization of switched neural networks via neural activation function and applications in image encryption. IEEE transactions on neural networks and learning systems, 26(2005), no. 7, 1493- 1502. doi: 10.1109/TNNLS.2014. 2387355.

[25] T. Hu, X. Zhang, S. Zhong. Global asymptotic synchronization of nonidentical fractional-order neural networks. Neurocomputing, 313(2018), 39-46. https://doi.org/10.1016/j.neucom.2018.05.098.

[26] W. Deng, C. Li, J. Lü. Stability analysis of linear fractional differential system with multiple time delays. Nonlinear Dynamics, 48(2007), no. 4, 409-416. https://doi.org/10.1007/s11071-006-9094-0.

[27] Y. Xu, J. Yu, W. Li. Global asymptotic stability of fractionalorder competitive neural networks with multiple time-varying-delay links. Applied Mathematics and Computation, 389(2021), 125498. https://doi .org/10. $1016 / j$.amc. $2020 \cdot 125498$. 
[28] A. Ouannas, F. Mesdoui, S. Momani. Synchronization of FitzHugh-Nagumo reaction-diffusion systems via one-dimensional linear control law. Archives of Control Sciences, 31(2021). doi: 10.24425/acs.2021.137421.

[29] A. Ouannas, I.M. Batiha, S. Bekiros, J. Liu, H. Jahanshahi. Synchronization of the glycolysis reactiondiffusion model via linear control law. Entropy, 23(2021), no. 11, 1516. https://doi.org/10.3390/ e23111516.

[30] M. Mellah, A. Ouannas, A.A. Khennaoui. Fractional Discrete Neural Networks with Different Dimensions: Coexistence of Complete Synchronization, Antiphase Synchronization and Full State Hybrid Projective Synchronization. Nonlinear Dynamics and Systems Theory, 21(2021), no. 4, 410. 